\title{
Piezo ion channels in cardiovascular mechanobiology
}

\author{
Dominique Douguet ${ }^{1}$, Amanda Patel ${ }^{1}$, Aimin $\mathrm{Xu}^{2}$, \\ Paul M. Vanhoutte ${ }^{2,3}$ and Eric Honoré ${ }^{*}$
}

1'Université Côte d'Azur, Centre National de la Recherche Scientifique, Institut National de la Santé et de la Recherche Médicale, Institut de Pharmacologie Moléculaire et Cellulaire, Labex ICST, Valbonne, France. ${ }^{2}$ State Key Laboratory of Biopharmaceutical Technologies, Department of Pharmacology and Pharmacy, The University of Hong Kong, Hong Kong, China. ${ }^{3}$ Department of Cardiovascular and Renal Research, Institute for Molecular Medicine, University of Southern Denmark, Odense, Denmark

*Correspondence: honore@ipmc.cnrs.fr

Key words: baroreflex, blood pressure, endothelium, Piezo1, shear stress, vascular smooth muscle cells

\begin{abstract}
Mechanotransduction plays a key role in vascular development, physiology and disease states. Piezo1 is a mechanosensitive non-selective cationic channel present in endothelial and vascular smooth muscle cells. It is activated by shear stress associated with increases in local blood flow, as well as by cell membrane stretch upon elevation of blood pressure. Here we briefly review the pharmacological modulators of Piezo and discuss the present state of knowledge on the role of Piezo1 in vascular mechanobiology and associated clinical disorders, such as atherosclerosis and hypertension. Ultimately, we believe that this recent research will help identify novel therapeutic strategies for the treatment of vascular diseases.
\end{abstract}

Blood flow generates a frictional force acting on the endothelium (shear stress, parallel to the vessel wall), as well as wall distension (stretch; a force perpendicular to the vessel wall) in response to changes in transmural pressure [1-4]. Shear stress can arise due to either laminar (smooth flow with fluid layers sliding in parallel) or turbulent (rough) flow of blood through the vasculature. These mechanical forces have a significant impact on vascular development, physiology and are implicated in various disease states, including atherosclerosis and hypertension [1-4]. Multiple mechanosensors (see Glossary) detect these mechanical forces within vascular cells, including elements of the extracellular matrix (ECM), adhesion molecules, the 
endothelium junctional complex, cytoskeletal components, G protein-coupled receptors [5], various ion channels [6-8] and a variety of signaling molecules, such as small GTPases and their effectors $[9,10]$.

Upon mechanical stimulation by fluid flow or membrane stretch, opening of mechanosensitive (MS) ion channels at the plasma membrane of cells is the earliest (within microseconds) cellular event [11-16]. These MS ion channels are characterized by different structures, ionic selectivity and gating properties [11-17]. Dimeric TREK/TRAAK $\mathrm{K}_{2 \mathrm{P}}$ channels are $\mathrm{K}^{+}$selective and their opening causes cell hyperpolarization in response to membrane stretch [18]. By contrast, trimeric Piezo1/2 cationic channels are non-selective and permeable to $\mathrm{Na}^{+}, \mathrm{K}^{+}$and $\mathrm{Ca}^{2+}$ and their activation in response to pressure stimulation (low threshold) or shear stress induces cell depolarization and $\mathrm{Ca}^{2+}$ influx [14, 19-21]. In addition, dimeric OSCA/TMEM63 non-selective cationic channels open in the high-pressure range (high threshold) [6, 22]. Here, we mainly focus on Piezo1, its role in cardiovascular mechanotransduction and properties as an emerging pharmacological target.

\section{Structural and functional properties of Piezo1}

Piezo ion channels are activated by mechanical stimuli such as localized membrane stretch, whole cell poking or fluid flow (i.e. shear stress) [14, 23]. Moreover, intracellular traction forces generated from myosin II phosphorylation by myosin light chain kinase are sufficient to generate spatially-restricted Piezo1-mediated $\mathrm{Ca}^{2+}$ flickers, in the absence of applied exogenous force (bioRxiv 294611; doi: https://doi.org/10.1101/294611). Piezo1 is present in peripheral tissues (including endothelial and arterial smooth muscle cells; ECs and SMCs), while Piezo2 (47\% identity with Piezo1) is highly expressed in mechanosensory neurons and responsible for light touch, proprioception, reflex regulation of breathing and baroreception (see below) (for review [14]). Notably, both Piezo1 and Piezo2 are coexpressed in the nodose and petrosal sensory neurons innervating the wall of the aorta and the carotid sinus [24]. Piezo1/2 ion channels are characterized by a fast inactivation mechanism (i.e. the amplitude of Piezo currents decreases with time, while mechanical stimulation is maintained), with a time constant $(\tau)$ of about 10 and $20 \mathrm{~ms}$ for Piezo2 and Piezo1 at $-80 \mathrm{mV}$, respectively) [19]. Mechanosensitivity is observed upon reconstitution of purified Piezo1 channels into artificial bilayers, indicating that the channel is a pore-forming subunit [25], directly activated by an increase in membrane tension [26]. While it was recently shown that Piezo1 flattens in the plane of the membrane in response to increased membrane tension [27 (the consequent increase in the in-plane channel cross sectional area is anticipated to provide the main driving energy controlling channel gating), currently only a closed state (or at least an inactive intermediate state) of Piezo1 is described \{Saotome, 2018 
\#2810, 28, 29](Figure 1A-B). Thus, the structure of the open state remains to be verified experimentally.

The amino terminal domain of Piezo1 is thought to form the mechanosensor (made of nine repeats forming the blades, each containing four transmembrane segments), while the $\mathrm{C}$ terminal domain constitutes the ionic pore lined by the last transmembrane segment (inner helix), the C-terminal extracellular domain (CED) and the intracellular C-terminal domain (CTD) [28-31](Figure 1A-B). Piezo1 is curved with the amino terminal domains resembling the three blades of a propeller and the ionic pore located at the central axis (Figure 1A-B). Notably, the insertion of Piezo1 into artificial liposomes causes a curvature of the membrane, forming a dome shape [29]. Piezo1 is predicted to deform the membrane into a "curved footprint" [32]. Thus Piezo1 may affect responses to curvature-sensitive molecules (for instance other MS ion channels) located in its vicinity [32].

Conformational changes in the $\mathrm{N}$ terminal domain caused by force may be transmitted to the central pore by decreasing the angle between an intracellular helix ("beam") beyond the channel and the membrane plane [33](Figure 1B). The main determinant of Piezo1 fast inactivation is an hydrophobic gate in the inner pore helix [34]. An unstructured domain located adjacent to the beam may act as a cytosolic plug that is thought to limit ion permeation possibly by clogging the inner pore of Piezo1/2 [35]. Of note, Piezo2 shares a similar trimeric structure with Piezo1, but shows an additional constriction site at the external side of the channel [36]. Whether or not a similar external constriction site is present in Piezo1 is unknown at this stage [28-31]. Thus, it remains possible that the available structures of Piezo1 may correspond to an intermediate inactive state. In some cell types, including ECs, native Piezo1 currents show a slow inactivation $[37,38]$. Thus, Piezo1 in ECs remains open longer while flow is maintained constant.

\section{Pharmacological Modulators of Piezo channels}

Over the years, a small number of pharmacological modulators of piezo channels were used in various studies. We briefly lay put some of them below. 
GsMTx4: GsMTx4 is a peptide toxin from Grammostola spatulata spider venom that blocks cationic stretch-activated channels [39]. It is a positively charged amphipathic molecule that can insert into lipid bilayers and inhibit various cationic MS channels, including Piezo1, TREK-1 and the bacterial channel MscL [40-42] (Figure 1B-C). Piezo1 currents are reversibly inhibited by GsMTx4 with a $\mathrm{K}_{\mathrm{d}}$ of $\sim 155 \mathrm{nM}$ [43]. Both enantiomeric forms of GsMTx4 inhibit Piezo1 [42]. Thus, GsMTx4 presumably acts by modulating local membrane tension near the MS channels and behaves as a gating modifier [39] (Figure 1B-C).

Yoda1 and Dooku1: The pharmacology of Piezo1 has further emerged with the identification of a small molecule activator called Yoda1 (Figure 1B, D) (EC50: 17.1 and $26.6 \mu \mathrm{M}$ for mouse and human Piezo1, respectively)[44]. It is important to note that Yoda1 sensitizes the channel to mechanical stimulation, but is not able to activate the channel by itself without applied force (leftward shift in the pressureeffect curve) [44]. A minimal region in Piezo1 [1961-2063] has been identified that is responsible for the activation by Yoda1 [45] (Figure 1A-B, magenta). Yoda1 enhances Piezo1 activation by pressure, but remarkably has no effect on the Piezo2 isoform [44, 45]. The effect of Yoda1 is highly dependent on the dichloro substituents and on the oxidation state of the thioether linker [44,46](Figure 1D-E). A Yoda1 analogue called Dooku1 reversibly antagonizes Yoda1 activation of Piezo1, but lacks a stimulatory effect [46](Figure 1D and Figure 2). One nitrogen shared by the pyrazine (Yoda1) and pyrrole (Dooku1) group also appears as an important feature for activity (Arrows in Figure 1D) [46]. These data suggest a pharmacophore that involves two mutually perpendicular planes, of which one plane is outlined by the dichlorophenyl ring and the other one is formed by the thiadiazole and pyrazine parallel rings (pyrrole group in the case of Dooku1) (Figure 1E).

Jedi1 and Jedi2: In addition, two low affinity water soluble chemical activators, Jedi1 and Jedi2 activate Piezo1 from the extracellular side (EC50: 200 and $158 \mu \mathrm{M}$ for mouse Piezo1, respectively)[33] (Figure 1B, 1F). Extracellular loops 15-16 [657-677] and 19-20 [870-921] within the blades are critically required for Piezo1 activation by Jedi1 and 2 (Figure 1A-B, yellow).

Gadolinium and ruthenium red: Further, gadolinium and ruthenium red (polycations) have also been used as non-specific blockers of native stretch-activated ion channels, as well as exogenous Piezo1 expressed in transfected cells [25, 47].

\section{Piezo1 is critically required for early vascular development}

Piezo1 is a key endothelial mechanosensor required for early vascular development [20, 21](Figure 3A-B, Key Figure). Between embryonic days E9 and 10.5, Piezo1 is expressed in the developing cardiovascular system, notably in the endothelium and endocardium [20, 21]. In the adult mouse, such expression persists in ECs, as well as in SMCs of resistance arteries)[48]. Piezo1 is also present in human umbilical vein and fetoplacental ECs [21, 46, 49]. 
Constitutive genetic invalidation of Piezo1 is embryonically lethal [20, 21]. Piezo1deficient embryos die at mid-gestation, despite normal initial vasculogenesis until E9.5, indicating that angiogenesis per se is not impaired. In Piezo1 knock-out mice, the yolk sac, as well as the embryonal vasculature is disorganized, lacking major blood vessels [20, 21]. Embryos lacking Piezo1 exhibit major pericardial effusion, reflecting a defective circulation and die before E14.5 [20, 21]. A similar phenotype in mice with endothelium-specific deletion of Piezo1 (with embryonic or early postnatal lethality) indicates that the defect in vascular architecture is caused by a lack of Piezo1 in ECs [21, 50].

\section{Piezo1 is an endothelial flow-activated non-selective cationic channel permeable to $\mathrm{Ca}^{2+}$}

Patch-clamp recordings have demonstrated the evidence of both pressure- and flowactivation of endothelial Piezo1, with a single channel conductance of about 25-30 pico Siemens (pS) [20, 21]. Stimulation of Piezo1 in ECs by either pressure or flow only exhibits a slow current inactivation (i.e. channels remain open longer in response to mechanical stress) and causes a steady increase in intracellular $\mathrm{Ca}^{2+}$ concentration [21]. Remarkably, transfection of Piezo1 in HEK 293 cells confers sensitivity to shear stress [21]. The opening of endothelial Piezo1-channels by shear stress activates downstream $\mathrm{Ca}^{2+}$-dependent calpains (proteolytic enzymes) which in turn are responsible for focal adhesion turnover, proteolytic cleavage of actin cytoskeletal elements and alignment of ECs to blood flow [20, 21](Figure 3A, Key Figure). In line with these findings, it has been shown that genetic deletion of Piezo1 or its inhibition with GsMTx4 suppresses shear stress-induced $\mathrm{Ca}^{2+}$-influx in ECs and impairs alignment of ECs to flow [20,21]. Denser actin structures are detected in Piezo1-depleted ECs and the channels accumulate at the leading apical lamellipodia, a site where focal adhesions turnover is enhanced upon alignment to flow [21]. In addition, opening of Piezo1 and the resulting $\mathrm{Ca}^{2+}$ influx in response to shear stress or to the pro-angiogenic factor sphingosine 1-phosphate $\left(\mathrm{S}_{1} \mathrm{P}\right)$, presumably in the absence of mechanical stimulation, causes the activation of the matrix metalloproteinase-2 (MMP-2) and membrane type 1 matrix metalloproteinase (MT1MMP) in ECs causing endothelial sprouting (Figure 3B, Key Figure) [51]. Thus, Piezo1 is critically required during early development for alignment and migration of ECs to blood flow, capillary network formation, determining number of vessel branch points and tube- length [20, 21, 51](Figure 2A-B, Key Figure). Likewise, in adult animals, conditional Piezo1 deletion in the endothelium impairs angiogenesis under conditions of wound injury or hindlimb ischemia [51].

\section{Piezo1 is required for morphogenesis of valves}

\section{Lymphatic valves}

Lymphatic vessels transport extravasated fluid back to the venous circulation, antigens and antigen-presenting cells to the lymph nodes and play a key role in the absorption of dietary fats [52]. The active propulsion of the lymph to the venous circulation occurs by synchronizing peristaltic waves and the opening/closing of 
intraluminal valves in the lymphatics [53]. The latter valves, originate from lymphatic ECs which develop into elongated valve leaflets following reorientation, active migration and matrix organization [54].

PIEZO1 recessive loss-of-function mutations cause congenial lymphatic dysplasia $[55,56]$, an inherited altered development of the lymphatic system that causes leakage of lymph fluid into the limbs. Moreover, in the mouse, specific invalidation of Piezo1 in ECs caused impaired lymphatic valve formation, leading to pleural effusion [50, 57]. Moreover, conditional Piezo1 invalidation in adult lymphatics caused substantial lymphatic valve degeneration [57]. Expression of the transcription factors forkhead box C2 (FoxC2) and nuclear factor of activated T cells 1 (NFATc1, implicated in the early induction of lymphatic valves), as well as the number of valve forming region were not altered upon Piezo1 deletion in ECs [50]. However, Piezo1 knockdown in cultured lymphatic ECs prevented the up-regulation of the lymphatic valve signature genes induced by oscillating shear stress [57]. Conversely, activation of Piezo1 by Yoda1 accelerated lymphatic valve formation in animals and also triggered upregulation of lymphatic valve genes in cultured lymphatic ECs in the absence of mechanical stimulation [57]. In the same line, activation of Piezo1 with Yoda1 induced remodeling of actomyosin and cell-cell adhesion sites in cultured lymphatic ECs [50]. In brief, collective migration, actin polymerization and remodeling of cell-cell junctions were impaired upon deletion of Piezo1 in ECs [50]. These findings clearly indicate that shear stress activation of Piezo1 is responsible for lymphatic valve morphogenesis [50,57].

\section{Arterial and venous valves}

Three rare PIEZO1 heterozygous variants, with minor allele frequencies $<1 \%$, have been found to be associated with bicuspid aortic valves in humans (bioRxiv 368365; doi: https://doi.org/10.1101/368365). Those variants (Y2022H, K2502R and S217L) produce inactive PIEZO1 channels and behave as dominant negative mutants when co-expressed with wild-type channels. Moreover, piezo1 has also been shown to be involved in the formation of arterial valves in zebrafish (bioRxiv 368365; doi: https://doi.org/10.1101/368365 and bioRxiv 528588; doi:https://doi.org/10.1101/528588).

In addition, Piezo1 is required for mouse venous valve formation [50]. In the same line, a single nucleotide polymorphism (SNP rs2911463) in PIEZO1 is associated with varicose veins of the lower extremities [58], but whether or not this variant affects channel function per se is unknown.

Ultimately, taken in conjunction, these findings suggest a key role for Piezo1 in the development of arterial, lymphatic and venous valves.

\section{Endothelial Piezo1 mediates flow-dependent vasodilatation and atheroprotection} Vasodilator factors such as nitric oxide (NO), released from the endothelium in response to increased blood flow contribute to the regulation of arterial blood 
pressure $[59,60]$. Shear stress activation of Piezo1 in mesenteric arteries releases ATP from ECs, partly through pannexin channels, which in turn stimulates P2Y2 purinergic receptors in an autocrine manner [61] (Figure 3C, Key Figure). The downstream Gq/G11-mediated activation of the MS complex PECAM-1, VE-cadherin and VEGFR2 enhances phosphorylation of endothelial nitric oxide synthase (eNOS) at Ser 1176 via PI3K/AKT, resulting in NO production [61](Figure 3C, Key Figure). In synergy, the increased flow induces, in a yet to define way, Piezo1-dependent release of adrenomedullin from the endothelium causing phosphorylation of eNOS at Ser 632 by PKA downstream of Gs-coupled CALCRL/RAMP2 receptors, thus further enhancing NO levels [62](Figure 3C, Key Figure). Increased NO levels in turn mediates vasodilatation (Figure 2 and Figure 3C, Key Figure).

Endothelial invalidation of Piezo1 at the adult stage (conditional knock-out) causes a blunted in vivo eNOS phosphorylation and decreased NO release possibly associated with an increased systolic blood pressure [61]. In mesenteric arteries or aortic rings of adult mice, conditional knock-out of Piezo1 in the endothelium prevents flow- or Yoda1-induced vasodilatation, although endothelium-dependent relaxations to acetylcholine, as well as endothelium-dependent contractions to the $\alpha_{1}$-adrenergic agonist, phenylephrine (PE) or the thromboxane-prostanoid (TP) receptor activator U46619 are preserved [46, 61](Figure 2). Similar findings were obtained with endothelial deletion of P2Y2, Gq/G11 or pannexin 1/2 [61]. In addition, mice with endothelial specific invalidation of adrenomedullin, adrenomedullin receptors or Gos also exhibit decreased release of NO in response to shear stress, impaired flowdependent vasodilatation and are hypertensive [62]. Treatment with Yoda1 also causes relaxation of murine intrapulmonary arteries in a Piezo1- and NO-dependent manner [63]. However, endothelial invalidation of Piezo1 does not influence the development of pulmonary hypertension in response to chronic hypoxia [63]. In rats, Piezo1 has also been shown to mediate flow-dependent vasodilatation of uterine arteries during pregnancy [64]. Accordingly, Yoda1 produces relaxation of preconstricted uterine arteries [64]. Finally, inhibition of Piezo1 expression in the endothelium by miR-103a is involved in acute myocardial infarction by influencing endothelium function [65].

Taken in conjunction, these data suggest that Piezo1-activation by shear stress regulates NO release by the endothelium, thereby influencing local vascular tone and, in turn, arterial blood pressure [20, 21, 46, 49, 61] and exerting an atheroprotective role (Figure 3C, Key Figure).

\section{Activation of Piezo1 by turbulent flow promotes atherogenic endothelial inflammation}

As discussed above, Piezo1 opening induced by laminar flow exerts an atheroprotective effect resulting from eNOS activation and NO release [2, 59, 6668](Figure 3C, Key Figure). However, at the inner (but not at the outer) curvature of the aortic arch where ECs are subjected to turbulent flow, Piezo1 activation induces 
endothelial inflammation and atherosclerosis [2, 59, 66-68]. Indeed, it has been observed that upon conditional endothelial Piezo1-deletion, the accumulation of endothelial inflammatory cells is reduced [69]. Moreover, endothelial deletion of Piezo1 or Gq/G11 in mice lacking low density lipoprotein (LDL) receptors and fed a high fat diet attenuates endothelial inflammation, as well as neointima formation after partial carotid ligation, causing atherosclerosis [69]. Integrin $\alpha 5$ and FAK activations are enhanced by turbulent flow in a Piezo1-/Gq/G11-dependent manner, causing stimulation of the NF-kB pathway and expression of pro-atherogenic genes [69](Figure 3D, Key Figure).

Collectively, these findings indicate that activation of Piezo1 by laminar flow is atheroprotective by eNOS stimulation [69](Figure 3C, Key Figure), while turbulent flow contributes to atherosclerosis through the NF-kB pathway (Figure 3D, Key Figure).

\section{Endothelial Piezo1 mediates exercise-induced mesenteric vasoconstriction}

Besides NO, additional relaxing factors, including prostacyclin, arachidonic acid metabolites, $\mathrm{H}_{2} \mathrm{O}_{2}, \mathrm{CO}_{2}, \mathrm{H}_{2} \mathrm{~S}$ and peptides are released by the endothelium, causing the opening of various $\mathrm{K}^{+}$channels in SMCs [70]. In addition, opening of the small and intermediate conductance $\mathrm{Ca}^{2+}$-activated $\mathrm{K}^{+}$channels $\left(\mathrm{SKCa}^{2+}\right.$ and $\mathrm{IKCa}^{2+}$, respectively) in the endothelium also causes hyperpolarization of electrically coupled SMCs through gap junctions (Figure 4A). In parallel, $\mathrm{K}^{+}$efflux (predominantly through endothelial $\mathrm{IKCa}^{2+}$ ) also activates Kir channels of SMCs (increase in single channel conductance) and/or stimulates their $\mathrm{Na}^{+} / \mathrm{K}^{+}$ATPase, also contributing to hyperpolarization and relaxation (Figure $4 \mathrm{~A}$ ). The resulting hyperpolarization at rest (i.e. without physical exercise), known as endothelium-dependent hyperpolarization (EDH) leads to relaxation (in this case of mesenteric arteries; Figure 4A). EDHdysfunction is involved in various disease states, including hypertension, and the various players acting in $\mathrm{EDH}$ are increasingly recognized as putative therapeutic targets [70].

By contrast, physical exercise-induced high blood flow enhances Piezo1 opening and causes depolarization of mesenteric artery ECs, due to the influx of $\mathrm{Na}^{+}$and $\mathrm{Ca}^{2+}$ (Figure 3E, Key Figure and Figure 4B). Depolarization is transmitted to the electrically coupled SMCs through gap junctions and triggers vasoconstriction mediated by the opening of nicardipine (dihydropyridine)-sensitive CaV1.2 channels (Figure 4B). EDH, that can be prevented by a mixture of the $\mathrm{K}^{+}$channel blockers apamin and charybdotoxin, is amplified in the mesenteric arteries of mice lacking endothelial Piezo1 [38]. Indeed, it has been shown that when mice were active on running wheels at night, invalidation of Piezo1 in ECs significantly lowered the elevated blood pressure [38]. Mice lacking endothelial Piezo1 exhibited reductions in distance runs, percentages of active time and number of active bouts of exercise [38]. Thus, endothelial Piezo1 plays a role in increasing blood pressure upon exercise and its opening in the endothelium by the high blood flow exerts an anti-EDH effect [38](Figure 3E and Figure 4B). However, it is important to note that the contribution 
of endothelial Piezo1 to flow-induced depolarization/constriction appears to be specific for the mesenteric circulation, as the EDH-mediated response is not amplified by Piezo1 invalidation in either saphenous or carotid arteries [38].

In summary, these findings demonstrate that endothelial Piezo1 activation by high blood flow associated with physical exercise contributes to the vasoconstriction of the mesenteric circulation (along with sympathetic activation), thereby optimizing physical performance [38](Figure 3E, Key Figure and Figure 4B).

\section{Piezo1 is involved in vascular hyperpermeability}

It has been shown that when lung microvessel-pressure is elevated by raising left atrial pressure or by aortic constriction, the resulting leakage of serum is abrogated by conditional endothelium specific invalidation of Piezo1 or its in vivo inhibition with GsMTx4 [71], thus indicating that pressure-induced opening of Piezo1 in ECs of lung microvessels mediates endothelial barrier disruption [71](Figure 3A, Key Figure). Pharmacological inhibition of calpain mimics the protective effect of Piezo1 invalidation [71], suggesting that Piezo1-opening results in calpain activation with subsequent degradation of the adherens junction proteins and leads to disintegration of the endothelial barrier (Figure 3A, Key Figure). Pulmonary edema which commonly occurs at high altitude (in people affected by mountain sickness), as well as during heart failure and after head trauma, is caused by leakage of plasma into the lung parenchyma when the endothelial barrier of lung capillaries is disrupted due to elevated hydrostatic pressure [72]. These findings suggest that pharmacological inhibition of Piezo1 might therefore be beneficial in the context of lung edema.

\section{Piezo1 in arterial SMCs mediates hypertensive arterial remodeling}

Expression of Piezo1 is strong in SMCs of resistance arteries, but sparse in conduit arteries [48]. Accordingly, patch clamp recordings reveal large stretch-activated nonselective (i.e. with a reversal potential close to $0 \mathrm{mV}$ ) cationic currents in myocytes that arise from resistance arteries, and little currents or not from conduit arteries [48]. Conditional deletion of Piezo1 in arterial SMCs fully suppress the MS currents in resistance artery myocytes [48]. Although myogenic constrictions induced by an increase in intraluminal pressure (of either mesenteric or cerebral arteries) are independent of smooth muscle Piezo1, the channel is involved in the arterial remodeling of resistance arteries during chronic hypertension [48](Figure 3F, Key Figure). Thus, unlike in normotensive animals, the deletion of SMC Piezo1 decreases arterial diameter, as well as media thickness under hypertensive conditions, resulting in a lower cross-sectional area [48].

Piezo1 opening causes an increase in cytosolic $\mathrm{Ca}^{2+}$-concentration which stimulates transglutaminase II (TGII) activity, an enzyme involved in the remodeling of the cytoskeleton, as well as of the ECM [48](Figure 3F, Key Figure). Indeed, it has been observed that in vivo inhibition of TGII by cystamine reverses Piezo1-dependent remodeling [48]. These findings establish a functional connection between Piezo1 
opening in vascular SMCs and a clinically relevant arterial remodeling that contributes to systemic hypertension.

\section{Role of Piezo1/2 in the baroreceptor reflex}

Upon an increase in arterial blood pressure, baroreceptor nerve endings in the wall of the aortic arch and carotid sinus are stretched triggering an afferent signal to the brain that stimulates the vagal nerve to slow down heart rate and reduces sympathetic tone to the vascular periphery, thereby allowing a normalization of blood pressure [73]. Dysfunction of the baroreflex is associated with premature death in patients suffering from postmyocardial infarction \{Stocker, 2019 \#2924.

Both Piezo1 and Piezo2 are expressed in the nodose-petrosal-jugular ganglion complex which includes the neuronal cell bodies of the baroreceptors \{Zeng, 2018 $\# 2803$. Genetic invalidation of both Piezo1 and Piezo2 in the nodose and petrosal sensory ganglia of mice fully impairs the baroreflex induced by an increase in blood pressure following phenylephrine (PE)-infusion [24]. Aortic depressor nerve activity is suppressed and the blood pressure variability of these double knock-out mice is enhanced [24]. Consequently, these show labile hypertension and increased blood pressure variability, recapitulating the clinical phenotype of human patients with baroreflex failure. However, it is interesting to note that invalidation of either Piezo1 or Piezo2 alone does not affect the baroreflex, compared to the controls [24]. Conversely, optogenetic activation of Piezo2-positive sensory afferents initiates the baroreflex [24]. These findings indicate that Piezo1 and Piezo2 work in concert on MS nerve endings of the baroreceptors, to modulate baroreceptor reflex.

\section{Conclusions and future perspectives}

The variety of the current research indicates that the Piezo1/2 cationic MS channels play a key role in multiple aspects of cardiovascular development and physiology and are involved in development of various disease states, including atherosclerosis and hypertension. Piezo1 is required for vasculogenesis during early development with a key function in the alignment of ECs to blood flow, sprouting and formation of endothelial tubes (Figure 3A-B, Key Figure) [20, 21]. Moreover, Piezo1 is involved in the formation of lymphatic, arterial and venous valves [50, 58]. Further, endothelial Piezo1 mediates flow-dependent vasodilatation of the mesenteric circulation by stimulating the release of NO [61], while vasoconstriction of the mesenteric circulation during physical exercise (Figure 3C and 3E, Key Figure) [38]. Notably, opening of Piezo1 by laminar blood flow is atheroprotective, while its activation by turbulent flow mediates endothelial inflammation [69](Figure 3C and E, Key Figure). Interestingly, stimulation of Piezo1 opening in lung capillaries by elevated hydrostatic pressure causes endothelial barrier disruption and edema (Figure 3A, Key Figure) [71]. Moreover, in SMCs of resistance arteries, Piezo1 contributes to hypertensive arterial remodeling [48] (Figure 3F, Key Figure). Finally, 
both Piezo1 and Piezo2 are required for proper baroreceptor function as their double invalidation causes labile hypertension and blood pressure variability [24].

Thus, the clinical phenotypes associated with genetic mutations in both human PIEZO1/2 genes, as well as the experimental phenotypes of various invalidation mouse models demonstrate the key importance of this class of MS ion channels. Although, the functional role of Piezo1/2 in cardiovascular pathophysiology is now well established, their pharmacology remains limited to low affinity drugs (activators and blockers) with poor solubility and stability that preclude in vivo usage [33, 44, 46](Figure 1). Thus, more work will be required to identify additional modulators.

Various outstanding questions also need to be addressed for a better understanding of the function and for improving the pharmacology of Piezo1:

- What is the 3-D structure of the Piezo1 open state? Recent structural (cryo electron microscopy of reconstituted Piezo1 in lipid vesicles) and functional (high speed atomic force microscopy) findings indicate that Piezo1 flattens upon activation and thereby affects local membrane curvature [27]. It is thus anticipated that Piezo1 gating might affect the function of neighboring proteins that are sensitive to membrane curvature, including other types of MS channels and/or membrane receptors [27, 29, 32]. Thus, it will be important to explore the collective behavior of different types of MS ion channels.

- Is Piezo1 activated by endogenous openers (i.e. "Yoda1-like" molecules) and/or inhibited by endogenous blockers (i.e. "GsMTx4-like" molecules)[43]? At this stage, only $\mathrm{S}_{1} \mathrm{P}$ was claimed to activate endothelial Piezo1, independently of force [51]. By contrast, the short chain saturated margaric fatty acid (conjugated to phospholipids), was shown to inhibit channel opening [74]. Moreover, cholesterol depletion was also reported to lower Piezo1 activation by membrane stretch (right shift of the pressureeffect curve) (bioRxiv 604488; doi: https://doi.org/10.1101/604488). Thus, lipids appear to be important endogenous modulators of Piezo1 opening and more work is required for a better understating of their role in vascular mechanobiology.

- Is the effect of shear stress on Piezo1 direct or does it involve an additional flow sensor? Does glycosylation of Piezo1 condition its flow activation? What is the role of the extracellular matrix in shear stress activation of Piezo1? Does Piezo1 interact with adhesion molecules and what is the impact on Piezo1 activation by force? Interestingly, recent findings indicate a partnership between Piezo1 and adhesion molecules mediating sub cellular tuning of force response (bioRxiv 602532; doi: https://doi.org/10.1101/602532).

- Why does native Piezo1 lack or show slow inactivation in specific cell types, including EC, mesenchymal stem cells and epithelial renal cells [21, 37, 75]? Whether or not Piezo1 variants possibly influencing inactivation are expressed in ECs is unknown at this stage.

- Are varicose veins associated with a dysfunctional Piezo1?

- Is Piezo1 expressed at the primary cilium of ECs and does it contribute to flowdependent dilatation? Previous findings indicated that the polycystins TRPP1/TRPP2 
at the primary cilium, facing the luminal side of ECs, mediate flow-dependent activation of the endothelium [76, 77]. Moreover, the TRPP1/TRPP2 ratio was shown to modulate the gating of cationic non-selective stretch-activated channels (presumably Piezo1) in arterial myocytes [75, 78]. Whether or not Piezo1 is targeted to the primary cilium of ECs and similarly modulated by polycystins is currently unknown.

- What is the role of Piezo1 in pulmonary artery remodeling? A role for Piezo1 in arterial myocytes was shown in hypertension-dependent arterial remodeling of resistance arteries of the general circulation [48]. Whether or not a similar role is at play in the pulmonary circulation needs to be investigated.

- Is Piezo1 a potential pharmacological target for the treatment of hypertension and/or atherosclerosis? Since Piezo1 is involved in hypertension-induced arterial remodeling, as well as atherosclerosis associated with turbulent flow, "blockers" might exert beneficial in these disease states. However, since Piezo1 is broadly expressed (including in red blood cells and immune cells [79-81], it is anticipated that inhibition of its function might cause important side effects.

- What is the pharmacology of Piezo2? It will be useful to identify both activators and inhibitors of the Piezo2 isoform that, besides its role in the baroreceptors, also plays a key role in touch sensing, proprioception and lung inflation-induced apnoea [82-87]. The high resolution structure of Piezo2 was recently solved and opens up novel opportunities for drug design [36].

-Besides the low threshold Piezo1/2 channels, the high threshold non-selective cationic MS channels OSCA/TMEM63 have also been recently discovered [6, 22, 8890]. What are the physiological roles of the TMEM63 isoforms in the cardiovascular system? What is their pharmacology?

-Although current research strongly suggests a role for Piezo1/2 in the baroreflex, there are still some concerns [73]. In particular, localization of Piezo1/2 within nerve terminal endings in the arterial wall is not yet demonstrated at the protein level (although optogenetic experiments suggest that it might well be the case [24]). Also, controls for nerve viability in the double Piezo1/2 knock-out mice are also required [24]. Moreover, the validity of blood pressure measurements was questioned, considering: i) low pulse pressure values (difference between systolic and diastolic blood pressure); ii) important delays between the increase in blood pressure induced by PE-infusion and the increase in aortic depressor nerve activity in the report by [24]; iii) requirement for very large increases in blood pressure to evoke the baroreflex; iv) absence of baseline blood pressure or heart rate values; v) lack of findings with decreased blood pressure [24, 73]. Thus, further experiments will be required to confirm a definitive role for Piezo1/2 as the baroreceptor transduction channels [24, 73]. Of note, other candidate ion channels, including the epithelial sodium channel or acid-sensing ion channels (ASIC), as well as TRPC5 are involved in the baroreflex [91-93] and have been proposed to act as MS channels in vascular cells. Whether or not those channels might work in concert (either upstream, 
downstream or in parallel) with Piezo1/2 is unclear at this stage and will need to be addressed in future studies.

In conclusion, the recent findings about structure-function relationships, genetic, physiological and pharmacological properties of Piezo1/2 advance in a major way our understanding of vascular mechanobiology. We hope that these will contribute in identifying novel therapeutic strategies for the treatment of major vascular disease states, including atherosclerosis and hypertension.

Acknowledgements: We thank F. Aguila for expert graphical help. We are grateful to Pr. David Beech, Pr. Stefan Offermanns and Pr. Ardem Patapoutian and for their valuable inputs on this manuscript. This work was supported by the Agence Nationale de la Recherche (ANR), France, The Fondation pour la Recherche Médicale (FRM), France and the Human Frontier Science Program (HFSP), France.

\author{
RESOURCES: \\ bioRxiv 294611; doi: https://doi.org/10.1101/294611 \\ bioRxiv 368365; doi: https://doi.org/10.1101/368365 \\ bioRxiv 528588; doi:https://doi.org/10.1101/528588 \\ bioRxiv 604488; doi: https://doi.org/10.1101/604488 \\ bioRxiv 602532; doi: https://doi.org/10.1101/602532
}

\title{
REFERENCES:
}

1. Mammoto, T. et al. (2013) Mechanobiology and developmental control. Annu Rev Cell Dev Biol 29, 27-61.

2. Hahn, C. and Schwartz, M.A. (2009) Mechanotransduction in vascular physiology and atherogenesis. Nat Rev Mol Cell Biol 10 (1), 53-62.

3. Davies, P.F. (1995) Flow-mediated endothelial mechanotransduction. Physiol Rev 75 (3), 519-60.

4. Davis, M.J. and Hill, M.A. (1999) Signaling mechanisms underlying the vascular myogenic response. Physiol Rev 79 (2), 387-423.

5. Xu, J. et al. (2018) GPR68 Senses Flow and Is Essential for Vascular Physiology. Cell 173 (3), 762-775 e16.

6. Murthy, S.E. et al. (2018) OSCA/TMEM63 are an Evolutionarily Conserved Family of Mechanically Activated Ion Channels. Elife 7, doi: 10.7554/eLife.41844.

7. Blondeau, N. et al. (2007) Polyunsaturated Fatty Acids Are Cerebral Vasodilators via the TREK-1 Potassium Channel. Circ Res 101, 176-84.

8. Garry, A. et al. (2007) Altered acetylcholine, bradykinin and cutaneous pressure-induced vasodilation in mice lacking the TREK1 potassium channel: the endothelial link. EMBO Rep $8(4), 354-9$.

9. Humphrey, J.D. et al. (2014) Mechanotransduction and extracellular matrix homeostasis. Nat Rev Mol Cell Biol 15 (12), 802-12.

10. Jaalouk, D.E. and Lammerding, J. (2009) Mechanotransduction gone awry. Nat Rev Mol Cell Biol 10 (1), 63-73. 
11. Christensen, A.P. and Corey, D.P. (2007) TRP channels in mechanosensation: direct or indirect activation? Nat Rev Neurosci 8 (7), 510-21.

12. Kung, C. (2005) A possible unifying principle for mechanosensation. Nature 436 (7051), 647-54.

13. Delmas, P. and Coste, B. (2013) Mechano-gated ion channels in sensory systems. Cell 155 (2), 278-84.

14. Murthy, S.E. et al. (2017) Piezos thrive under pressure: mechanically activated ion channels in health and disease. Nat Rev Mol Cell Biol 18 (12), 771-783.

15. Hamill, O.P. and Martinac, B. (2001) Molecular basis of mechanotransduction in living cells. Physiol. Rev. 81, 685-740.

16. Sachs, F. (2010) Stretch-activated ion channels: what are they? Physiology (Bethesda) 25 (1), 50-6.

17. Nilius, B. and Honore, E. (2012) Sensing pressure with ion channels. Trends Neurosci 35 (8), 477-86.

18. Honoré, E. (2007) The neuronal background K2P channels: focus on TREK-1. Nature reviews neuroscience 8, 251-61.

19. Coste, B. et al. (2010) Piezo1 and Piezo2 are essential components of distinct mechanically activated cation channels. Science 330 (6000), 55-60.

20. Ranade, S.S. et al. (2014) Piezo1, a mechanically activated ion channel, is required for vascular development in mice. Proc Natl Acad Sci U S A 111 (28), 10347-52.

21. Li, J. et al. (2014) Piezo1 integration of vascular architecture with physiological force. Nature 515, 279-282.

22. Zhang, M. et al. (2018) Structure of the mechanosensitive OSCA channels. Nat Struct Mol Biol 25 (9), 850-858.

23. Wu, J. et al. (2017) Touch, Tension, and Transduction - The Function and Regulation of Piezo Ion Channels. Trends Biochem Sci 42 (1), 57-71.

24. Zeng, W.Z. et al. (2018) PIEZOs mediate neuronal sensing of blood pressure and the baroreceptor reflex. Science 362 (6413), 464-467.

25. Coste, B. et al. (2012) Piezo proteins are pore-forming subunits of mechanically activated channels. Nature 483, 176-181.

26. Syeda, R. et al. (2016) Piezo1 Channels Are Inherently Mechanosensitive. Cell Rep 17 (7), 1739-1746.

27. Lin, Y.C. et al. (2019) Force-induced conformational changes in PIEZO1. Nature 573, 230234.

28. Zhao, Q. et al. (2018) Structure and mechanogating mechanism of the Piezo1 channel. Nature 554 (7693), 487-492.

29. Guo, Y.R. and MacKinnon, R. (2017) Structure-based membrane dome mechanism for Piezo mechanosensitivity. Elife 6, doi: 10.7554/eLife.33660.

30. Ge, J. et al. (2015) Architecture of the mammalian mechanosensitive Piezo1 channel. Nature 527 (7576), 64-9.

31. Saotome, K. et al. (2018) Structure of the mechanically activated ion channel Piezo1. Nature 554 (7693), 481-486.

32. Haselwandter, C.A. and MacKinnon, R. (2018) Piezo's membrane footprint and its contribution to mechanosensitivity. Elife 7, doi: 10.7554/eLife.41968.

33. Wang, Y. et al. (2018) A lever-like transduction pathway for long-distance chemical- and mechano-gating of the mechanosensitive Piezo1 channel. Nat Commun 9 (1), 1300. 
34. Zheng, W. et al. (2019) A hydrophobic gate in the inner pore helix is the major determinant of inactivation in mechanosensitive Piezo channels. Elife 8, doi: 10.7554/eLife.44003.

35. Taberner, F.J. et al. (2019) Structure-guided examination of the mechanogating mechanism of PIEZO2. Proc Natl Acad Sci U S A.

36. Wang, L. et al. (2019) Structure and mechanogating of the mammalian tactile channel PIEZO2. Nature 573, 225-229.

37. Del Marmol, J.I. et al. (2018) Piezo1 forms a slowly-inactivating mechanosensory channel in mouse embryonic stem cells. Elife 7, doi: 10.7554/eLife.33149.

38. Rode, B. et al. (2017) Piezo1 channels sense whole body physical activity to reset cardiovascular homeostasis and enhance performance. Nat Commun 8 (1), 350.

39. Gnanasambandam, R. et al. (2017) GsMTx4: Mechanism of Inhibiting Mechanosensitive Ion Channels. Biophys J 112 (1), 31-45.

40. Bode, F. et al. (2001) Tarantula peptide inhibits atrial fibrillation. Nature 409 (6816), 35-6.

41. Suchyna, T.M. et al. (2000) Identification of a peptide toxin from Grammostola spatulata spider venom that blocks cation-selective stretch-activated channels. J Gen Physiol 115 (5), 583-98.

42. Suchyna, T.M. et al. (2004) Bilayer-dependent inhibition of mechanosensitive channels by neuroactive peptide enantiomers. Nature 430 (6996), 235-40.

43. Bae, C. et al. (2011) The mechanosensitive ion channel Piezo1 is inhibited by the peptide GsMTx4. Biochemistry 50 (29), 6295-300.

44. Syeda, R. et al. (2015) Chemical activation of the mechanotransduction channel Piezo1. Elife 4, doi: 10.7554/eLife.07369.

45. Lacroix, J.J. et al. (2018) Probing the gating mechanism of the mechanosensitive channel Piezo1 with the small molecule Yoda1. Nat Commun 9 (1), 2029.

46. Evans, E.L. et al. (2018) Yoda1 analogue (Dooku1) which antagonizes Yoda1-evoked activation of Piezo1 and aortic relaxation. Br J Pharmacol 175 (10), 1744-1759.

47. Yang, X.C. and Sachs, F. (1989) Block of stretch-activated ion channels in Xenopus oocytes by gadolinium and calcium ions. Science 243 (4894 Pt 1), 1068-71.

48. Retailleau, K. et al. (2015) Piezo1 in Smooth Muscle Cells Is Involved in HypertensionDependent Arterial Remodeling. Cell Rep 13 (6), 1161-1171.

49. Morley, L.C. et al. (2018) Piezo1 channels are mechanosensors in human fetoplacental endothelial cells. Mol Hum Reprod 24 (10), 510-520.

50. Nonomura, K. et al. (2018) Mechanically activated ion channel PIEZO1 is required for lymphatic valve formation. Proc Natl Acad Sci U S A 115 (50), 12817-12822.

51. Kang, H. et al. (2019) Piezo1 mediates angiogenesis through activation of MT1-MMP signaling. Am J Physiol Cell Physiol 316 (1), C92-C103.

52. Randolph, G.J. et al. (2017) The Lymphatic System: Integral Roles in Immunity. Annu Rev Immunol 35, 31-52.

53. Scallan, J.P. et al. (2016) Lymphatic pumping: mechanics, mechanisms and malfunction. J Physiol 594 (20), 5749-5768.

54. Semo, J. et al. (2016) Development of the lymphatic system: new questions and paradigms. Development 143 (6), 924-35.

55. Fotiou, E. et al. (2015) Novel mutations in PIEZO1 cause an autosomal recessive generalized lymphatic dysplasia with non-immune hydrops fetalis. Nat Commun 6, 8085.

56. Lukacs, V. et al. (2015) Impaired PIEZO1 function in patients with a novel autosomal recessive congenital lymphatic dysplasia. Nat Commun 6, 8329. 
57. Choi, D. et al. (2019) Piezo1 incorporates mechanical force signals into the genetic program that governs lymphatic valve development and maintenance. JCI Insight 4 (5).

58. Shadrina, A.S. et al. (2019) Varicose veins of lower extremities: Insights from the first large-scale genetic study. PLoS Genet 15 (4), e1008110.

59. Vanhoutte, P.M. et al. (2009) Endothelial dysfunction and vascular disease. Acta Physiol (Oxf) 196 (2), 193-222.

60. Zhou, J. et al. (2014) Shear stress-initiated signaling and its regulation of endothelial function. Arterioscler Thromb Vasc Biol 34 (10), 2191-8.

61. Wang, S. et al. (2016) Endothelial cation channel PIEZO1 controls blood pressure by mediating flow-induced ATP release. J Clin Invest 126 (12), 4527-4536.

62. Iring, A. et al. (2019) Shear stress-induced endothelial adrenomedullin signaling regulates vascular tone and blood pressure. J Clin Invest 130 (129), 2775-2791.

63. Lhomme, A. et al. (2018) Stretch-Activated Piezo1 Channel in Endothelial Cells Relaxes Mouse Intrapulmonary Arteries. Am J Respir Cell Mol Biol 60, 650-658.

64. John, L. et al. (2018) The Piezo1 cation channel mediates uterine artery shear stress mechanotransduction and vasodilation during rat pregnancy. Am J Physiol Heart Circ Physiol 315 (4), H1019-H1026.

65. Huang, L. et al. (2016) MiR-103a targeting Piezo1 is involved in acute myocardial infarction through regulating endothelium function. Cardiol J 23 (5), 556-562.

66. Gimbrone, M.A., Jr. and Garcia-Cardena, G. (2016) Endothelial Cell Dysfunction and the Pathobiology of Atherosclerosis. Circ Res 118 (4), 620-36.

67. Chiu, J.J. and Chien, S. (2011) Effects of disturbed flow on vascular endothelium: pathophysiological basis and clinical perspectives. Physiol Rev 91 (1), 327-87.

68. Davies, P.F. et al. (2005) Shear stress biology of the endothelium. Ann Biomed Eng 33 (12), 1714-8.

69. Albarran-Juarez, J. et al. (2018) Piezo1 and Gq/G11 promote endothelial inflammation depending on flow pattern and integrin activation. J Exp Med 215 (10), 2655-2672.

70. Feletou, M. and Vanhoutte, P.M. (2017) Endothelium-dependent hyperpolarizations: Quo vadis? Acta Physiol (Oxf) 219 (1), 100-107.

71. Friedrich, E.E. et al. (2019) Endothelial cell Piezo1 mediates pressure-induced lung vascular hyperpermeability via disruption of adherens junctions. Proc Natl Acad Sci U S A 116 (26), 12980-12985.

72. West, J.B. and Mathieu-Costello, O. (1995) Vulnerability of pulmonary capillaries in heart disease. Circulation 92 (3), 622-31.

73. Stocker, S.D. et al. (2019) Missing Pieces of the Piezo1/Piezo2 Baroreceptor Hypothesis: An Autonomic Perspective. J Neurophysiol 122, 1207-1212.

74. Romero, L.O. et al. (2019) Dietary fatty acids fine-tune Piezo1 mechanical response. Nat Commun 10 (1), 1200.

75. Peyronnet, R. et al. (2013) Piezo1-dependent stretch-activated channels are inhibited by Polycystin-2 in renal tubular epithelial cells. EMBO Rep 14 (12), 1143-8.

76. Aboualaiwi, W.A. et al. (2009) Ciliary Polycystin-2 Is a Mechanosensitive Calcium Channel Involved in Nitric Oxide Signaling Cascades. Circ Res 104 (7), 860-869.

77. Nauli, S.M. et al. (2008) Endothelial cilia are fluid shear sensors that regulate calcium signaling and nitric oxide production through polycystin-1. Circulation 117 (9), 1161-71.

78. Sharif-Naeini, R. et al. (2009) Polycystin-1 and -2 dosage regulates pressure sensing. Cell 139 (3), 587-96. 
79. Albuisson, J. et al. (2013) Dehydrated hereditary stomatocytosis linked to gain-of-function mutations in mechanically activated PIEZO1 ion channels. Nat Commun 4, 1884.

80. Cahalan, S.M. et al. (2015) Piezo1 links mechanical forces to red blood cell volume. Elife 4, doi: 10.7554/eLife.07370.

81. Solis, A.G. et al. (2019) Mechanosensation of cyclical force by PIEZO1 is essential for innate immunity. Nature 573 (7772), 69-74.

82. Maksimovic, S. et al. (2014) Epidermal Merkel cells are mechanosensory cells that tune mammalian touch receptors. Nature 509 (7502), 617-21.

83. Murthy, S.E. et al. (2018) The mechanosensitive ion channel Piezo2 mediates sensitivity to mechanical pain in mice. Sci Transl Med 10 (462).

84. Nonomura, K. et al. (2017) Piezo2 senses airway stretch and mediates lung inflationinduced apnoea. Nature 541 (7636), 176-181.

85. Ranade, S.S. et al. (2014) Piezo2 is the major transducer of mechanical forces for touch sensation in mice. Nature 516 (7529), 121-5.

86. Woo, S.H. et al. (2015) Piezo2 is the principal mechanotransduction channel for proprioception. Nat Neurosci 18 (12), 1756-62.

87. Woo, S.H. et al. (2014) Piezo2 is required for Merkel-cell mechanotransduction. Nature 509 (7502), 622-6.

88. Jojoa-Cruz, S. et al. (2018) Cryo-EM structure of the mechanically activated ion channel OSCA1.2. Elife 7, doi: 10.7554/eLife.41845.

89. Liu, X. et al. (2018) Structure of the hyperosmolality-gated calcium-permeable channel OSCA1.2. Nat Commun 9 (1), 5060.

90. Yuan, F. et al. (2014) OSCA1 mediates osmotic-stress-evoked Ca2+ increases vital for osmosensing in Arabidopsis. Nature 514 (7522), 367-71.

91. Drummond, H.A. et al. (1998) A molecular component of the arterial baroreceptor mechanotransducer. Neuron 21 (6), 1435-41.

92. Lu, Y. et al. (2009) The ion channel ASIC2 is required for baroreceptor and autonomic control of the circulation. Neuron 64 (6), 885-97.

93. Lau, O.C. et al. (2016) TRPC5 channels participate in pressure-sensing in aortic baroreceptors. Nat Commun 7, 11947.

\section{FIGURE LEGENDS}

\section{Figure 1: Structure and pharmacological modulators of Piezo1}

Piezo1 (PDB 6B3R; ribbon representation) is made of a trimer of subunits with the ionic pore located at the center of the triskelion. Shown here are the top (A) and the side (B) view of the 3-D atomic structure of Piezo1 (presumably in closed or inactive state) where each monomer is colored in blue, light gray and dark gray respectively. The dashed red lines illustrate the curvature of the molecule (i.e. membrane plane). B also shows the interactions of different pharmacological modulators of piezo1: GsMTx4, Yoda1, and Jedi1. The molecular surface of these small molecules is colored by the color of its closest atom. The critical region in Piezo1 required for Yoda1 activation [1961-2063] and the N-terminal extracellular loops ([657-677] and [870921]) required for Jedi1/2 activation are shown in magenta and yellow respectively. The structures and pharmacological molecules are at scale. (C) Structure of GsMTx4 
(hydrophobic residues in green and positively charged residues in blue). The primary sequence of the GsMTx4 sequence is:

Gly-Cys-Leu-Glu-Phe-Trp-Trp-Lys-Cys-Asn-Pro-Asn-Asp-

Asp-Lys-Cys-Cys-Arg-Pro-Lys-Leu-Lys-Cys-Ser-Lys-Leu-

Phe- Lys-Leu-Cys-Asn-Phe-Ser-Phe $\mathrm{NH}_{2}$

\section{(Disulfide bridges: $2-17,9-23$ and 16-30)}

(D) Yoda1 and its analogue, Dooku1 are shown. Dooku1 prevents Piezo1 activation by Yoda1 in the micromolar range. (E) Pharmacophore features of Yoda1 family of compounds resulting from available structure-activity relationships analyses, are illustrated. Orange circles and lines indicate pharmacophore points and molecular geometry. The pharmacophore involves two mutually perpendicular planes, of which one plane is outlined by the dichlorophenyl ring and the other one is formed by the thiadiazole and pyrazine parallel rings (pyrrole group in the case of Dooku1). (F) Jedi1 and Jedi2 are water soluble small molecule Piezo1 activators acting in the millimolar range within the blade domains of Piezo1 (in yellow panels A-B).

\section{Figure 2: Endothelial Piezo1 mediates vasorelaxation}

Thoracic aortas from Piezo1 ${ }^{+/+}$mice were contracted by the addition of PE (acting on SMCs) [46]. Vasorelaxation was induced by the addition of Yoda1, that activates Piezo1 in ECs [46]. Similar findings were observed with rat uterine arteries [64]. Moreover, in pre-contracted mesenteric arteries from Piezo1 ${ }^{+/+}$mice, increased intraluminal flow or Yoda1 induced relaxation [61]. Yoda1-induced aorta relaxation was inhibited by Dooku1 (a Yoda1 analogue that does not activate Piezo1) [46]. Notably, removing the endothelium or conditional deletion of Piezo1 prevented relaxation by either Yoda1 or increased intraluminal fluid flow [46, 61].

Figure 3 (Key Figure): Piezo1 in vascular mechanotransduction. (A) Activation of Piezo1 by shear stress (laminar flow: blue arrow) during early development is required for alignment of ECs to flow through a $\mathrm{Ca}^{2+}$-dependent activation of calpain and cleavage of focal adhesion molecules. Opening of Piezo1 by high hydrostatic pressure $(\mathrm{P}$, red arrow) in lung capillaries causes disruption of endothelial adherens junctions by $\mathrm{Ca}^{2+}$-dependent activation of calpain, ultimately leasing to endothelial barrier disruption (associated with lung edema). (B) Activation of Piezo1 by laminar flow or sphingosine 1 phosphate $\left(S_{1} P\right)$ (presumably in the absence of mechanical stress) regulates the activation and membrane translocation of membrane type-1 matrix metalloproteinase (MT1-MMP), inducing ECs sprout formation. (C) Laminar blood flow induces vasodilatation through Piezo1 activation and release of NO (phosphorylation of eNOS S1176 and S632) and is atheroprotective. (D) Turbulent flow is atherogenic by a Piezo1-dependent stimulation of the NFkB pathway. (E) Activation of endothelial Piezo1 by high blood flow upon physical exercise induces depolarization of mesenteric artery SMCs through gap junctions, activates CaV1.2 causing calcium entry and induces vasoconstriction. (F) Opening of Piezo1 in SMCs of resistance arteries plays a role in arterial remodeling through stimulation of 
transglutaminase II. In C-D, atheroprotective and atherogenic processes are depicted in blue and red boxes respectively.

Figure 4: Endothelial Piezo1 exerts an anti EDH effect. (A) At rest (low blood flow) Piezo1 opening within the endothelium of mesenteric arteries is limited and EDH is associated with relaxation. Increase in endothelial $\mathrm{Ca}^{2+}$ in response to acetylcholine stimulation of the muscarinic M3 receptor promotes the opening of both $\mathrm{IKCa}^{2+}$ and $\mathrm{SKCa}^{2+}$. The resulting hyperpolarization is transmitted to SMCs through gap junctions. Hyperpolarization causes relaxation by closing of CaV1.2 channels (membrane potential is below the voltage threshold of CaV1.2 activation). (B) Upon physical exercise causing high blood flow in the mesenteric circulation, opening of endothelial Piezo1 leads to cell depolarization due to the influx of $\mathrm{Na}^{+}$and $\mathrm{Ca}^{2+}$. Depolarization is transmitted to electrically coupled SMCs through gap junctions (shown by yellow rectangles). Activation of CaV1.2 by cell depolarization causes a $\mathrm{Ca}^{2+}$ influx that triggers contraction of the myocytes. Piezo1 activation in the endothelium by high blood flow (i.e. shear stress) antagonizes EDH (endotheliumdependent hyperpolarization).

\section{GLOSSARY}

Adherens junction: protein complex at cell-cell junctions.

Angiogenic factor: a molecule that plays a role in blood vessel formation.

Baroreceptor: mechanosensory neurons specialized in sensing changes in blood pressure.

Cell depolarization: positive change in the membrane potential.

Channel inactivation : closing of the channel despite a maintained stimulation (mechanical in the case of Piezo channels).

Enantiomer: is one of two stereoisomers that are mirror images of each other nonsuperposable.

Focal adhesions: macromolecular complex containing integrins that establish a link between the cytoskeleton (inside the cell) and the extracellular matrix (ECM).

Ionic selectivity: the type of ion flowing through a channel

Lamellipodia: actin projection at the leading edge of a cell.

Lymph node: are major sites of B and T lymphocytes and other white blood cells.

Mechanosensor: a molecule specialized in sensing force.

Neointima: scar tissue that forms on the wall of blood vessels following lesions or injury.

Nitric oxide (NO): vasodilator gas produced by the endothelium in response to acetylcholine or bradykinin stimulation.

Optogenetic: biological technique that uses light to control various biological processes. 
Pannexins: large transmembrane channels (similar to gap junction hemichannels) connecting the intracellular and extracellular space, allowing the passage of ions and small molecules between these compartments, including ATP.

Patch-clamp: electrophysiological technique allowing for ion channel recordings. Peristaltic wave: smooth muscle cells contract in sequence to produce a wave allowing propulsion of lumen content.

Pharmacophore: represents the spatial arrangement of features that is essential for a molecule to interact with a specific target (Piezo1 in the present case). Purinergic receptor: a membrane receptor activated by ATP.

Resistance artery: small diameter artery controlling local blood flow. Shear stress: force vector component parallel to the wall of the artery. Single channel conductance: A measure of the size of the ionic pore. 
Figure 1

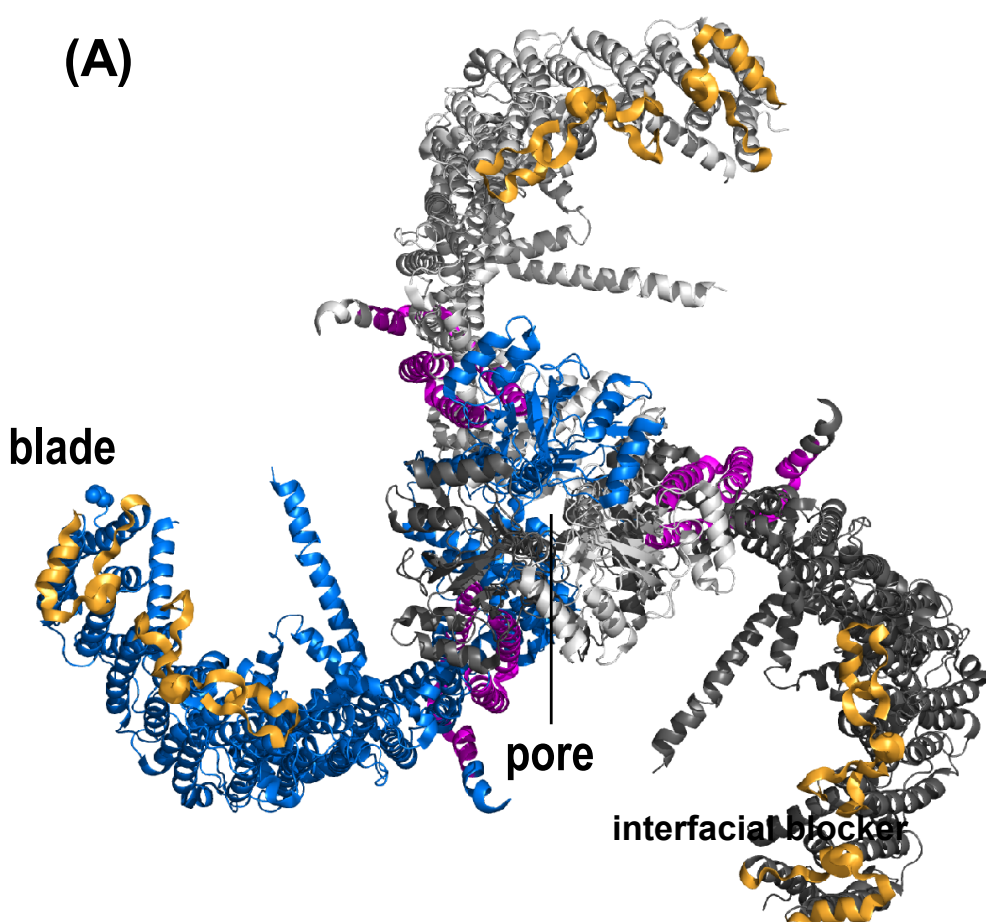

(B)

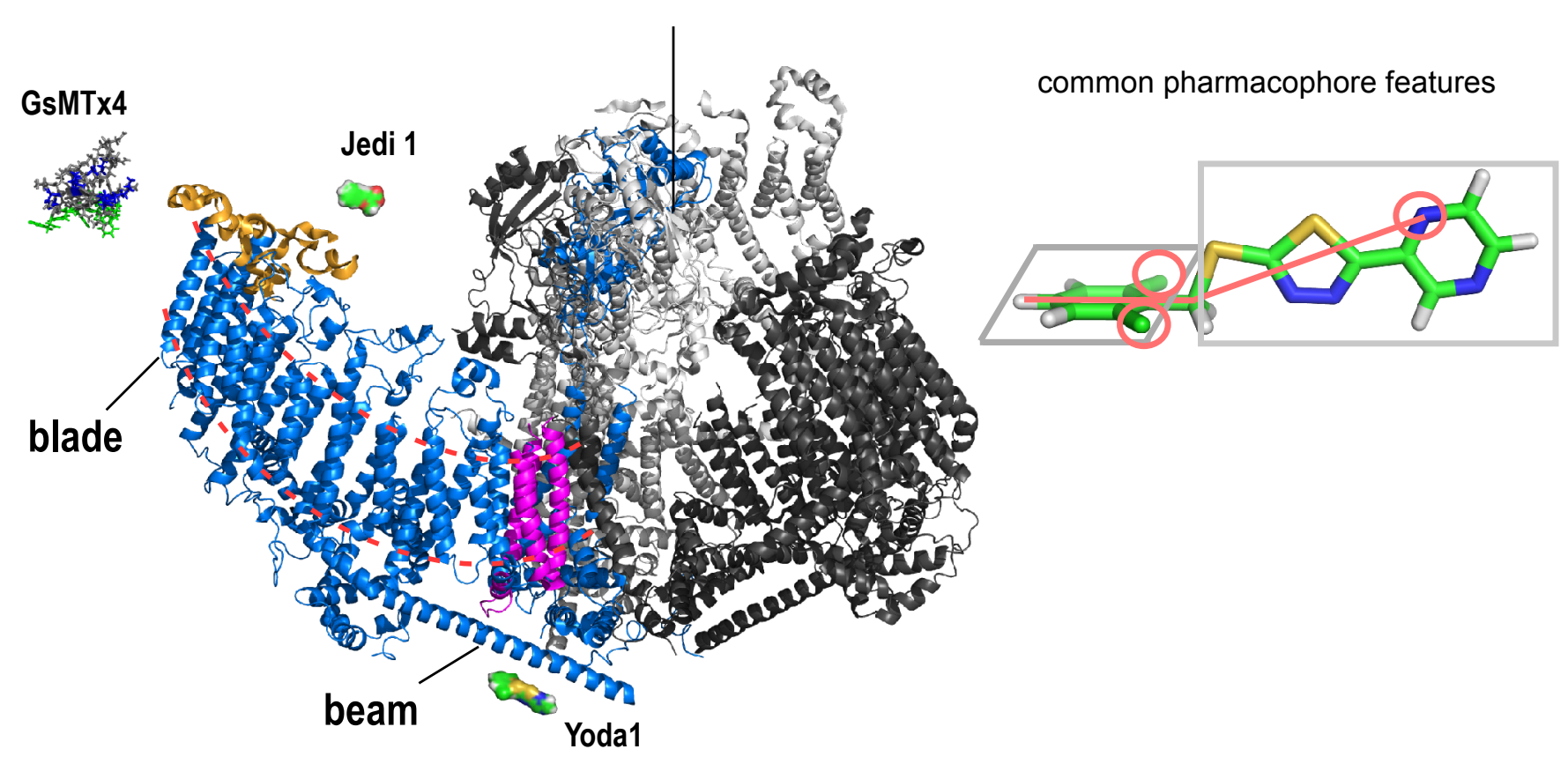

(D) Yoda1 (activator)
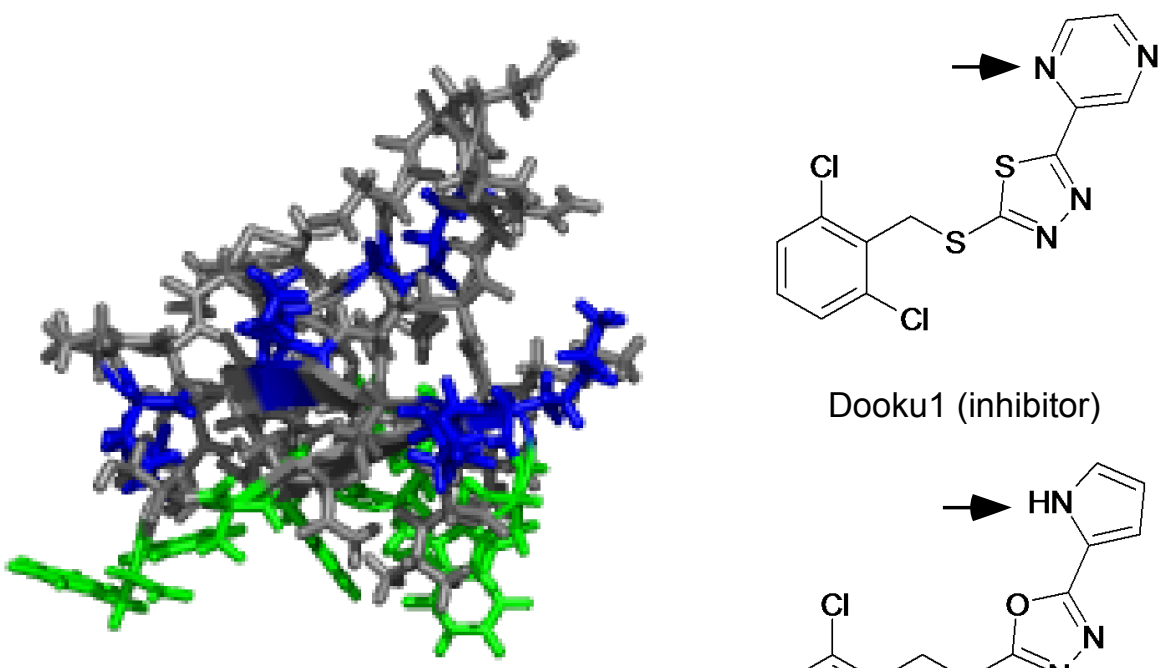

Dooku1 (inhibitor)

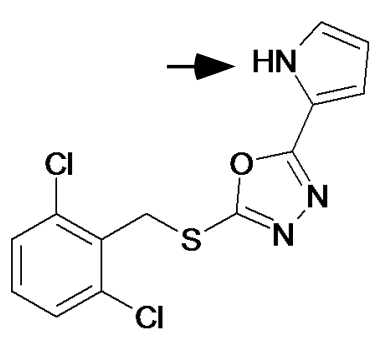

(F)

Jedi1 (activator)<smiles>Cc1oc(-c2ccccc2)cc1C(=O)O</smiles>

Jedi2 (activator)

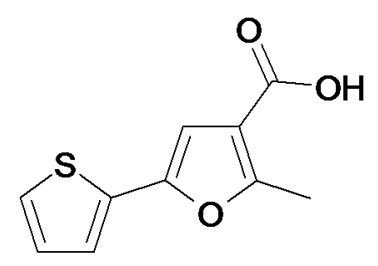


Figure 2

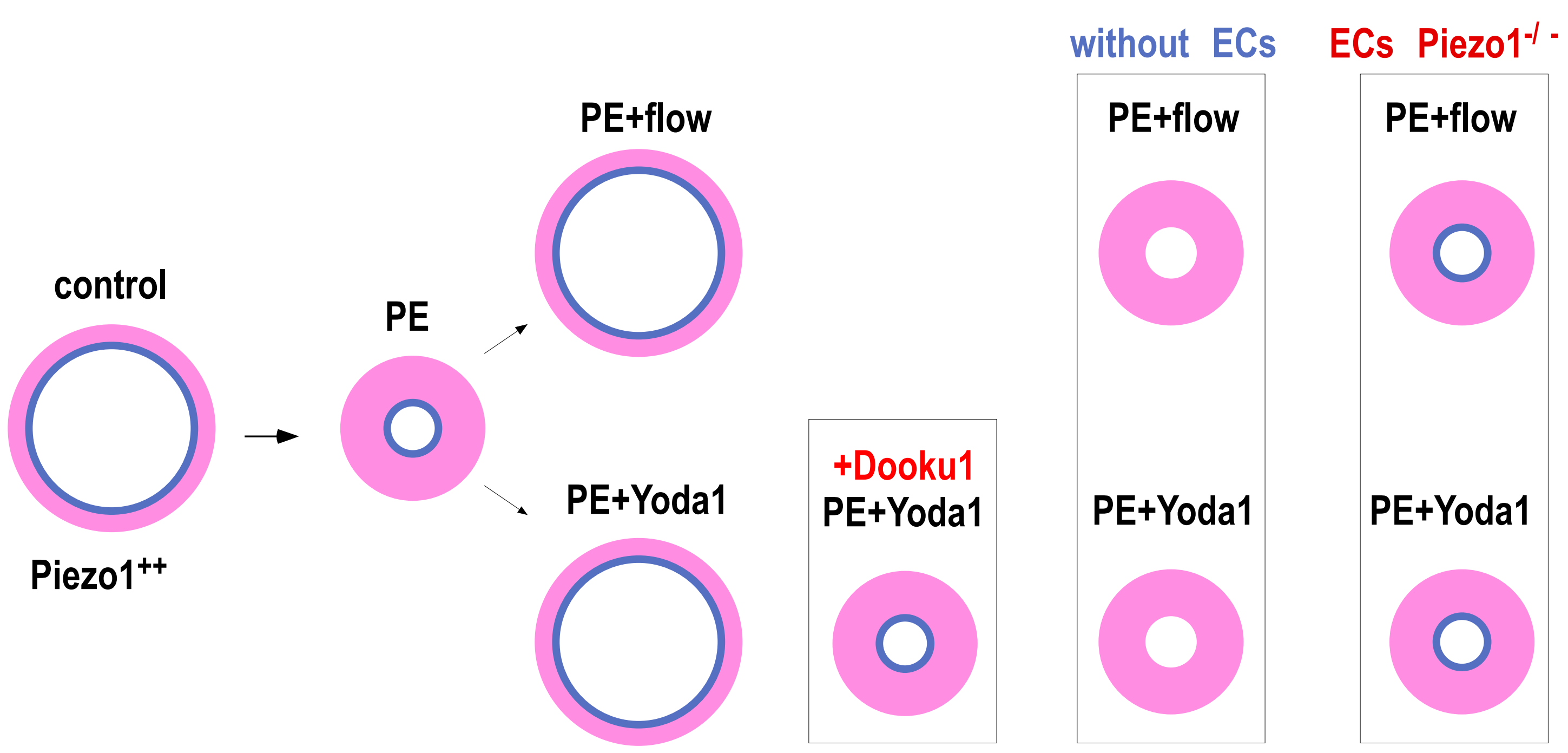


Figure 3, Key Figure

(A)

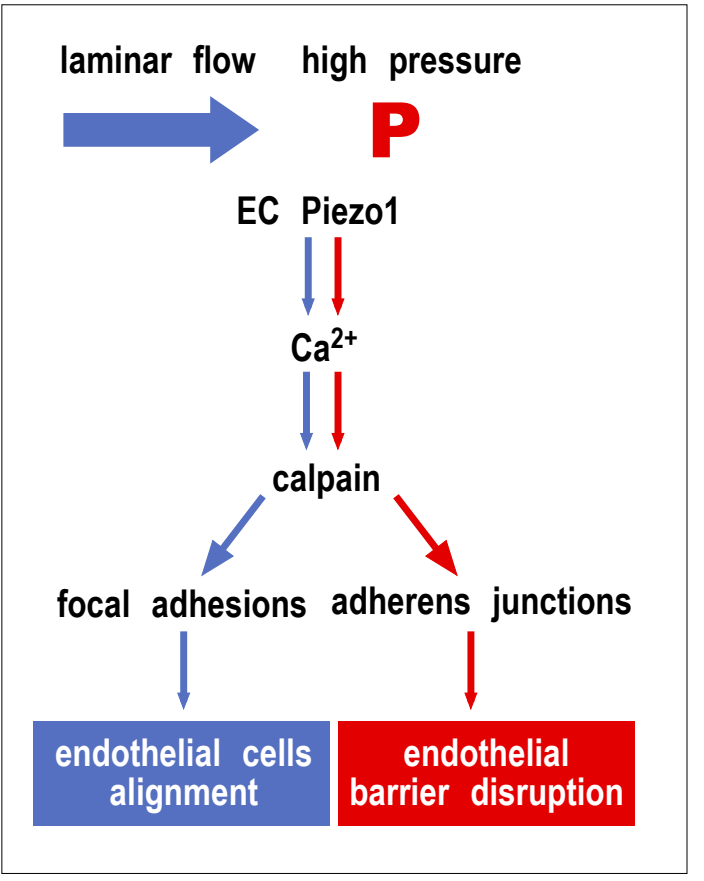

(D)

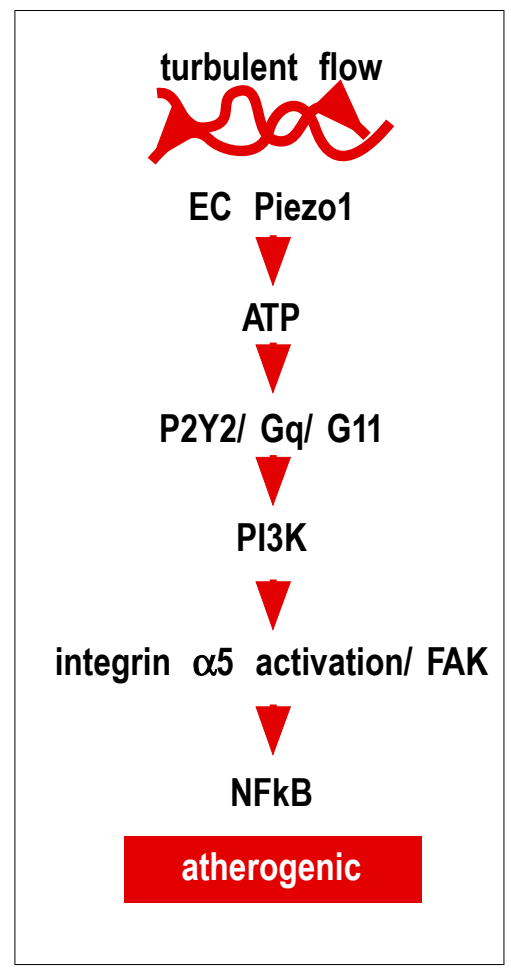

(B)

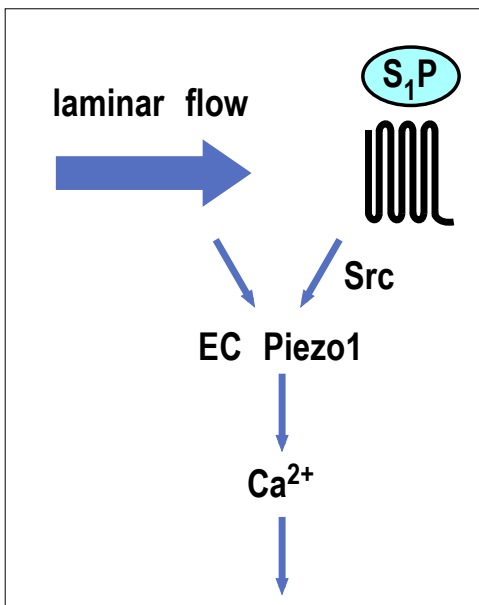

Pro MT1-MMP

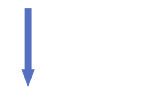

Active MT1-MMP

membrane translocation

endothelial cells sprout formation

(E)

physical exercise high flow

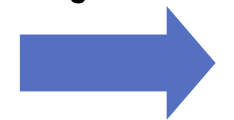

EC Piezo1

depolarization

SMC CaV1.2

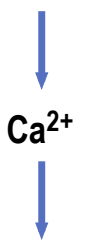

vasoconstriction
(C)

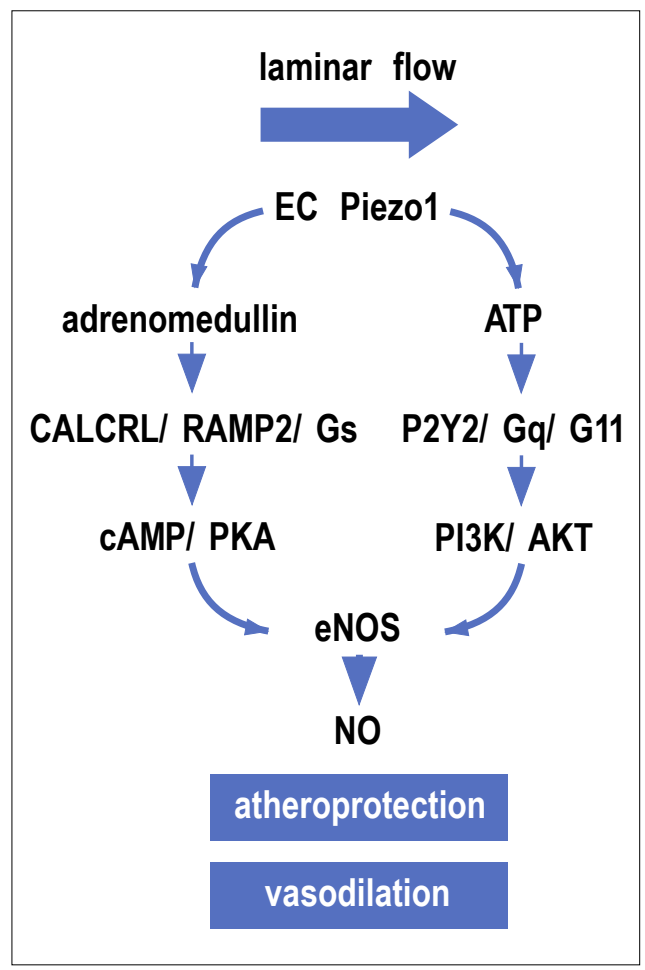

(F)

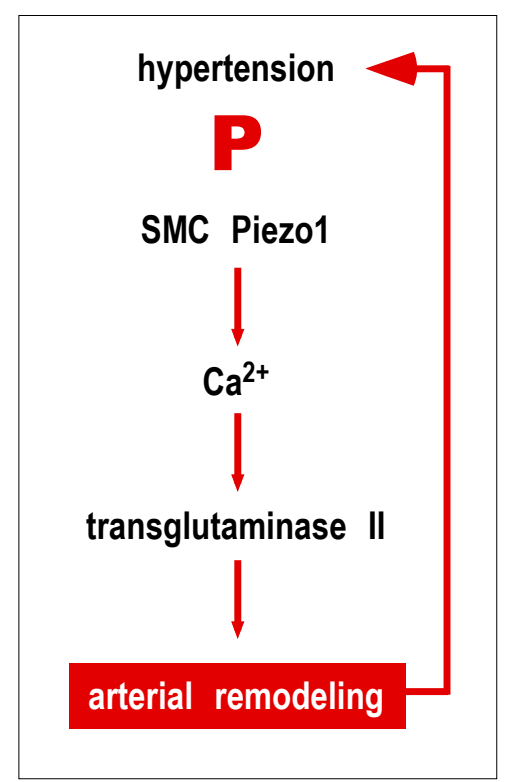


(A)

\section{REST}

hyperpolarization dilatation

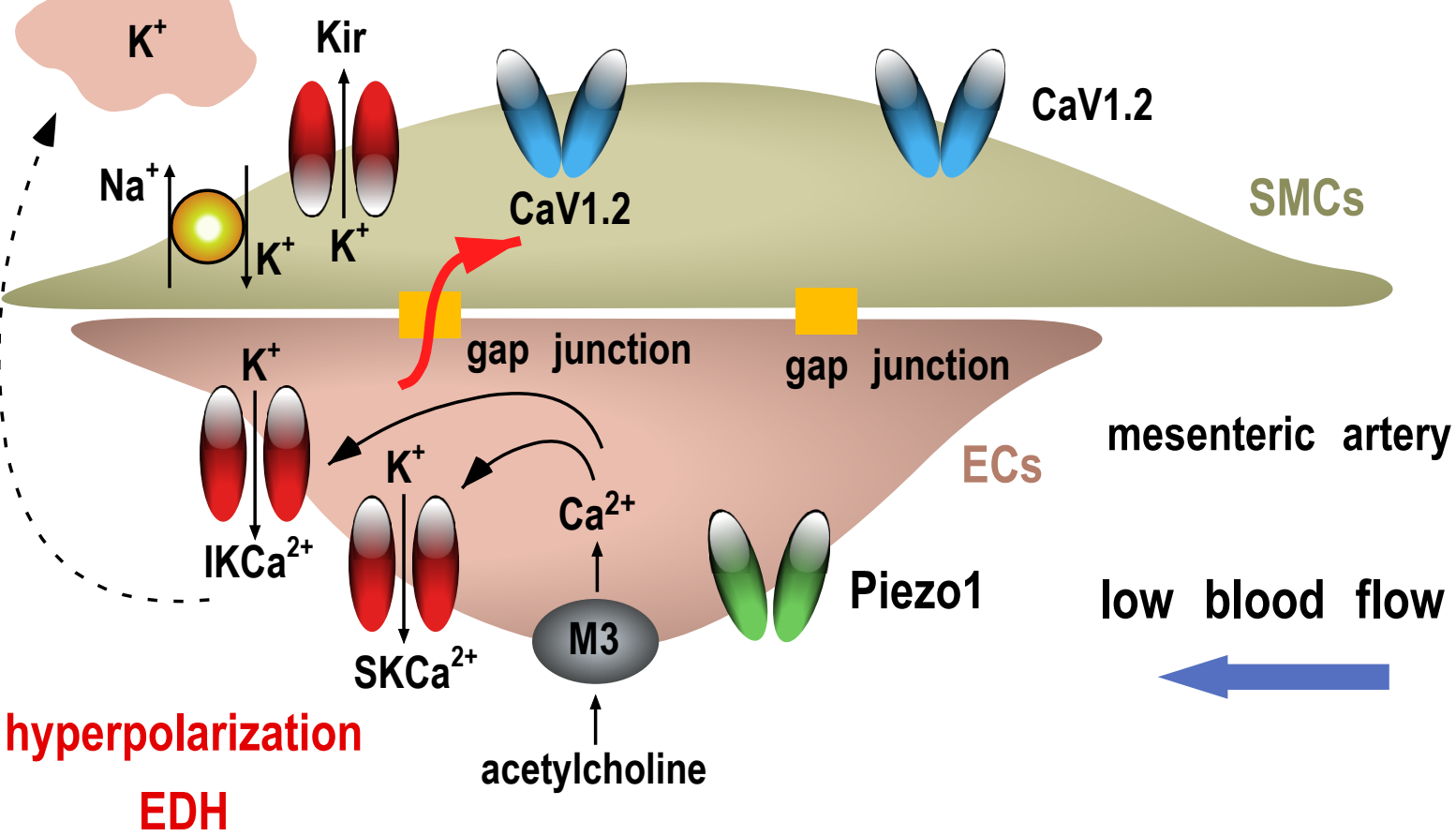

(B)

EXERCISE

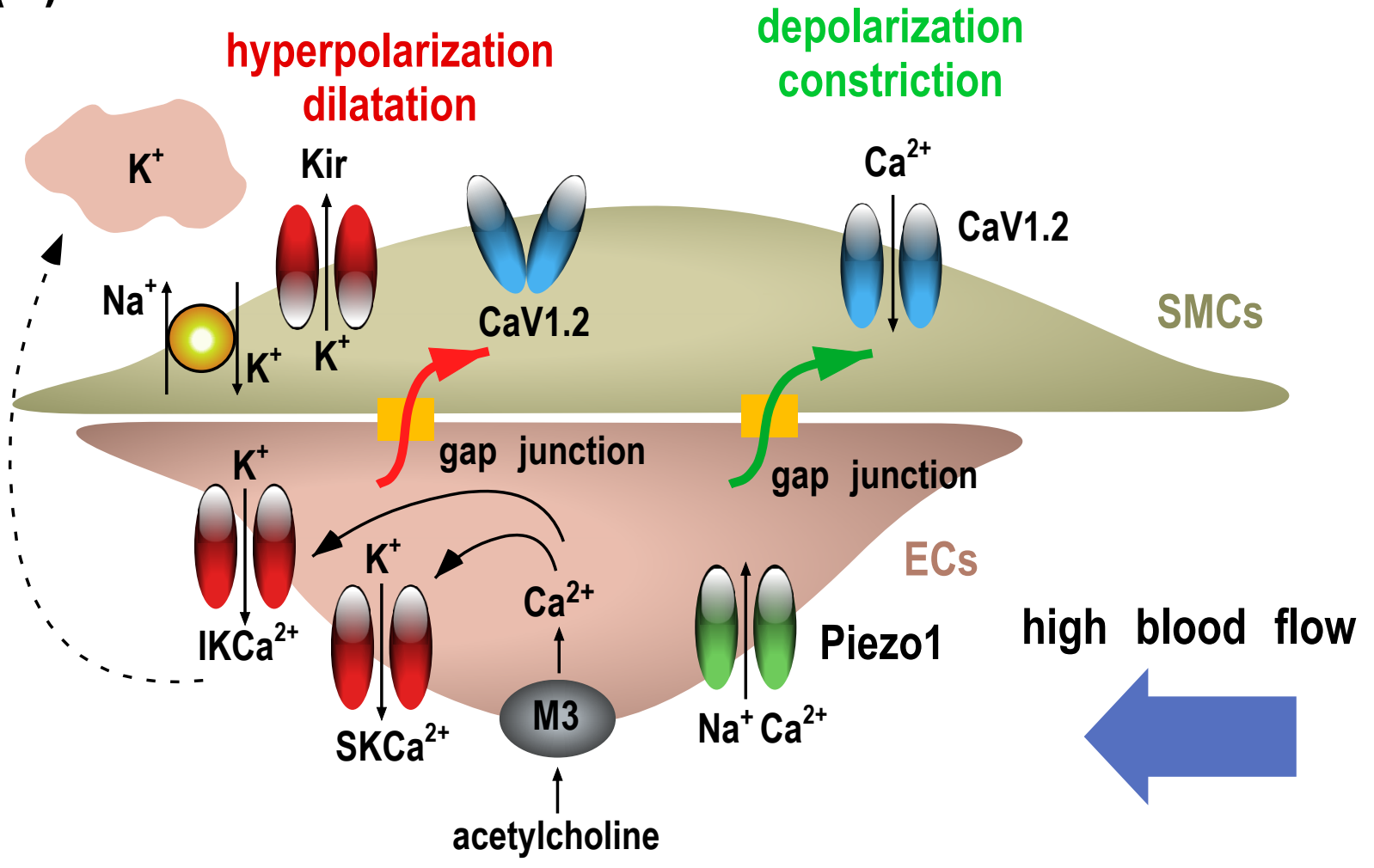

hyperpolarization

depolarization 